\title{
Formally Verifiable Modeling of In-Vehicle Time-Sensitive Networks (TSN) Based on Logic Programming
}

\author{
Morteza Hashemi Farzaneh, Sina Shafaei and Alois Knoll \\ Robotics and Embedded Systems \\ Technische Universität München \\ Boltzmannstr. 3, 85748 Garching bei München \\ Email:\{hashemif,shafaei,knoll\}@in.tum.de
}

\begin{abstract}
Increasing number of in-vehicle sensors, actuators and controllers involved in novel applications such as autonomous driving, requires new communication technologies to fulfill heterogeneous non-functional requirements such as latency, bandwidth and reliability. Time-Sensitive Networking (TSN) is a set of new standards in development by Institute of Electrical and Electronics Engineers (IEEE) defined to support mixed criticality based on Ethernet technology. This technology has recently raised significant attention of automotive domain. However, the mutual influence of application requirements in relation to TSN standards still remains a complex problem to master. For instance, considering an existing complex automotive network, an engineer has to carefully analyze the possible effects of adding new sensors on other existing critical applications. The network has to be configured such that the fulfilling of all requirements is verified. Targeting this problem, a modeling approach based on Logic Programming (LP) is developed to support more efficient configuration and verification process with focus on in-vehicle TSN networks.
\end{abstract}

\section{INTRODUCTION}

Time-Sensitive Networking (TSN) [1] is a set of new standards which are in developing process by Institute of Electrical and Electronics Engineers (IEEE) to support mixed criticality based on Ethernet technology. These standards will help to overcome challenging requirements of automotive domain considering upcoming innovative applications such as autonomous driving and infotainment that require e.g. fully deterministic network behavior, high bandwidth, failoperational and etc.

Despite the strengths of TSN, increasing number of involved sensors, actuators and Electronic Control Units (ECU) and mutual influence of requirements still cause high network engineering overhead for automotive network engineers. For example, considering an existing in-vehicle network and its applications' requirements, reviewing the whole configuration is required when new critical nodes join the network. After reviewing the TSN standards, some parts of the network configuration may need to be modified and after this reconfiguration one has to formally verify that all requirements are satisfied.

In this paper, a modeling approach based on Logic Programming (LP) is developed for configuration and verification of TSN networks. The major advantage of our approach compared to the other modeling approaches is that the whole model consists of logical facts and rules. Using inference algorithms such as backward chaining (e.g. used in Prolog), the complexity of verification of application requirements is reduced to build the correct queries on the model in order to verify specific network properties including non-functional requirements of the applications. The modeling approach is also used to find out interesting correlations between different requirements which are important for configuration. The main contributions are: definition of the logical facts and rules (work in progress) in section III and modeling demonstration using a concrete example in section IV including use case examples for configuration and verification.

\section{RELATED WORK}

An abstract methodology for modeling and verification of Cyber-Physical Systems (CPS) is developed and demonstrated in [2], [3], [4] using logic programming. The focus is to build a bridge between logic programming and hybrid automata as the underlying model with infinite structures and properties. In contrast to these contributions, we additionally deal with mutual influence of different requirements and configuration aspects of In-vehicle TSN. Declarative networking is proposed in [5], based on logic programming language Datalog which is a subset of Prolog. This contribution is extended in [6] in order to apply it for declarative network verification of e.g. routing protocols without discussing the configuration aspects. A practical declarative network management approach based on Datalog is presented in [7]. This paper only describes how to specify QoS requirements such as latency, jitter and bandwidth but it does not explain how to use it for configuration and verification. It has been shown in [8] that Prolog is a programming language which is sufficiently expressive and well-suited for the implementation of distributed protocols. In this paper, Prolog is applied for implementation of the TSN modeling approach. Simulation-based approaches [9], [10], [11], [12] are developed to analyze the performance of Audio Video Bridging (AVB) and TSN. The main disadvantage of these approaches is that not all of the corner cases can be 
covered by simulations and therefore are not suitable for required formal verification of critical requirements. In contrast, formal analysis methods are developed in [13], [14] to verify the performance of the TSN shapers. These methods however, focus only on timing and latency aspects on the network layer and do not respond to the question of mutual influence of critical requirements.

\section{A. Discussion}

The majority of the logic programming-based approaches focus on specification and verification of network protocols. We exploit logic programming for step-by-step modeling TSN features.

\section{MODELING APPROACH}

Prolog (SWI implementation) is used as the logical modeling language. It is restricted to Horn clauses and consists of facts and rules. Each rule has the form $\alpha:-\beta_{1}, \beta_{2}, \ldots, \beta_{n}$ that is equivalent to $\beta_{1} \wedge \beta_{2} \wedge \ldots \beta_{n} \Rightarrow \alpha . \alpha$ is called head and $\beta_{1}, \beta_{2}, \ldots, \beta_{n}$ is the body of the rule. It is obvious that head is true if the body is true. Each $\beta$ in the body is a call to a defined predicate. These predicates in the body are called goals and can be either a fact (a clause with empty body) or a rule.

Each PL-based TSN Model is a knowledge base that consists of Prolog facts and rules. Facts are used to describe properties and requirements in the network. The rules are used to describe the relations between facts and other rules. For instance,

publishes (front_camera, front_camera_p1, dom_cam [t_front_camera]).

is a fact describing that the device front_camera uses the Ethernet port front_camera_pl to send a data topic $t$ front_camera in the camera domain dom_cam and

firstPort (Dom, T, P) :- publishes (_, P, Dom, TL), member (T, TL) .

is a rule that declares that $P$ is the starting Ethernet egress port while transmitting data topic $T$ in domain Dom, if $(\{\Leftarrow\} \equiv\{:-\})$ a device publishes a list of data topics $T L$ in domain Dom using port $P$ and (and $\equiv \wedge \equiv\{$,$\} ) also T$ is a member of $T L$.

In the following, facts and rules are presented that are developed to model a TSN network step-by-step.

\section{A. Facts}

The modeling clauses consist of the following facts: topic, qos, publishes, consumes, device, switch and isLinked.

Each data topic $T$ is either a periodic topic or event-based topic. We define:

$$
\begin{gathered}
T_{\text {topic }}^{\text {periodic }}=\left(T_{\text {domain }}, T_{\text {name }}, T_{\text {periodic }}, T_{\text {period }}^{(\mu s)}, T_{\text {size }}^{(\text {Byte })}\right) \\
T_{\text {topic }}^{\text {event }}=\left(T_{\text {domain }}, T_{\text {name }}, T_{\text {event }}, T_{\text {size }}^{(\text {Byte })}\right)
\end{gathered}
$$

This classification of topics in periodic and event-based is significant regarding the hard real-time quality of service requirements. Data topics with tight timing requirements have to be modeled as periodic in order to calculate a feasible schedule with e.g. the time-aware shaper in IEEE 802.1Qbv. The combination of a topic domain and topic name is assumed to be unique in the whole TSN network. Consider all available topics: $T_{\text {topics }}=\left\{t_{1}, t_{2}, \ldots, t_{n}\right\}$ and all available domains as $T_{\text {domains }}=\left\{d o m_{1}, d o m_{2}, \ldots, d o m_{t}\right\}$. It holds that

$$
\forall_{T^{\prime}, T^{\prime \prime} \in T_{\text {topics }}}\left(T_{\text {domain }}^{\prime}=T_{\text {domain }}^{\prime \prime} \Rightarrow T_{\text {name }}^{\prime} \neq T_{\text {name }}^{\prime \prime}\right)
$$

The origin of the idea of using domains is in data-centric middleware approaches.

TSN nodes are either a device or a switch that consist of a set of Ethernet ports. Devices and switches use these ports to transmit or forward data topics embedded in Ethernet frames. Device and switch facts are defined as a tuple of name $D_{\text {name }}, S_{\text {name }}$ and the list of their ports $D^{\text {ports }}, S^{\text {ports }}$ as a subset of all existing ports in the network defined as $E P=\left\{p_{1}, p_{1}, \ldots, p_{n}\right\}$. Formally defined:

$$
\begin{gathered}
D_{\text {device }}=\left(D_{\text {name }}, D^{\text {ports }}\right), S_{\text {switch }}=\left(S_{\text {name }}, S^{\text {ports }}\right), \\
D_{\text {devices }}=\left\{D_{1}, D_{2}, \ldots, D_{m}\right\}, S_{\text {devices }}=\left\{S_{1}, S_{2}, \ldots, S_{t}\right\}, \\
\forall D_{i} \forall S_{j}\left(D_{i} \in D_{\text {devices }} \wedge S_{j} \in S_{\text {devices }}\right), \\
\bigcap_{i=1}^{m} D_{i}^{\text {ports }} \bigcap_{j=1}^{t} S_{j}^{\text {ports }}=\emptyset, \\
\bigcup_{i=1}^{m} D_{i}^{\text {ports }} \bigcup_{j=1}^{t} S_{j}^{\text {ports }}=E P
\end{gathered}
$$

Each device can publish or consumes a set of topics in a specific domain. The facts are formally defined as:

$$
\begin{gathered}
P_{\text {publishes }}^{\left\{T_{\text {topic }}, D_{\text {device }}\right\}}=\left(D_{\text {name }}, D_{\text {port }}, T_{\text {domain }}, P_{\text {topics }}^{\left\{t_{1}, t_{2}, \ldots, t_{u}\right\}}\right), \\
C_{\text {consumes }}^{\left\{T_{\text {topic }}, D_{\text {device }}\right\}}=\left(D_{\text {name }}, D_{\text {port }}, T_{\text {domain }}, P_{\text {topics }}^{\left\{t_{1}, \ldots, t_{v}\right\}}\right), \\
\quad\left\{t_{1}, t_{2}, \ldots, t_{u}\right\} \subseteq T_{\text {topics }},\left\{t_{1}, t_{2}, \ldots, t_{v}\right\} \subseteq T_{\text {topics }}
\end{gathered}
$$

The quality of service requirements of a topic among publisher and consumer devices are modeled using the fact qos which is defined as:

$$
\begin{gathered}
Q_{\text {qos }}^{\left\{T_{\text {topic }}, D_{\text {device }}\right\}}=\left(Q_{\text {type }}^{\{\text {latency,reliability }, \ldots\}}, T_{\text {domain }}, T_{\text {name }},\right. \\
\left.D_{\text {publisher }}, D_{\text {consumer }}, Q_{V L A N}^{\{0,1, \ldots, 7\}}\right)
\end{gathered}
$$

Network connections are defined using isLinked fact which has to be symmetric in order to describe bi-directional communication. Formally:

$$
\begin{gathered}
L_{\text {isLinked }}^{\left\{S_{\text {switch }}, D_{\text {device }}\right\}}=\left(L_{\text {port }}^{\prime}, L_{\text {port }}^{\prime \prime}, L_{\text {bandwidth }}^{\left(\frac{\text { Byte }}{s}\right)}\right), \\
\forall L_{\text {port }}^{\prime} \\
\forall L_{\text {port }}^{\prime \prime}\left(\exists D_{\text {device }}\left(L_{\text {port }}^{\prime} \in D^{\text {ports }}\right) \vee\right. \\
\left.\exists S_{\text {switch }}\left(L_{\text {port }}^{\prime \prime} \in S^{\text {ports }}\right)\right)
\end{gathered}
$$

\section{B. Rules (WORK IN PROGRESS)}

The modeling inference rules are developed step-by-step in order to obtain useful information based on the defined facts. Such information is used for simplification of network verification and configuration. To make the isLinked fact symmetric, link is defined as: 


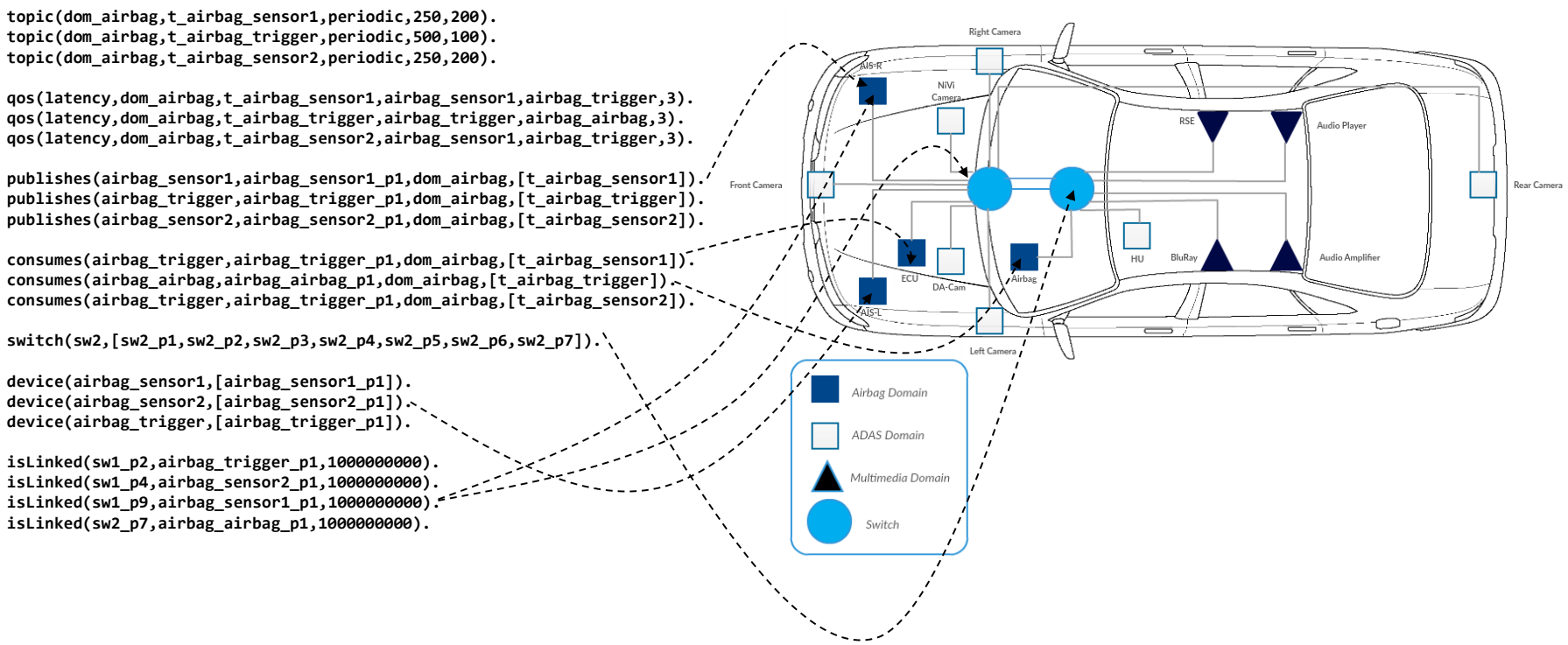

Fig. 1. An excerpt of the LP-based model of in-vehicle network using the formally defined facts. Using inference rules, interesting properties of the network can be verified.

It would return false if the number of disjoint paths is increased to $N>=3$.

The second example deals with the importance of egress port $s w 2 \_7$ for airbag trigger. It has to be verified that there is no data transmission of priority $\{$ vlan $=3\}$. The verification query in Prolog is:

?- streamPorts $\left({ }_{-}, \mathrm{T},,_{-}\right.$, Egress Ports , $)$, member (

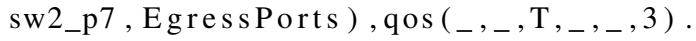

Prolog responds with false which means that there is definitely no disruptive communication on this port.

\section{CONCLUSION AND FUTURE WORK}

A network modeling approach based on logic programming is presented that afterwards is used to assist automotive network engineers, during the configuration and verification process. The uniqueness of this approach is that the whole network model is based on logical facts and rules which leads to more efficient configuration and verification process that normally costs a lot of engineering effort. This work is still in progress and we are extending the rules to model deep details of TSN with application in automotive domain.

\section{REFERENCES}

[1] "Time-Sensitive Networking." [Online]. Available: http://www.ieee802.org/1/pages/tsn.html

[2] G. Gupta and E. Pontelli, "A constraint-based approach for specification and verification of real-time systems," in Proceedings Real-Time Systems Symposium, Dec 1997, pp. 230-239.

[3] N. Saeedloei and G. Gupta, "A logic-based modeling and verification of cps," SIGBED Rev., vol. 8, no. 2, pp. 31-34, Jun. 2011. [Online]. Available: http://doi.acm.org/10.1145/2000367.2000374

[4] — , "A methodology for modeling and verification of cyberphysical systems based on logic programming," SIGBED Rev., vol. 13, no. 2, pp. 34-42, Apr. 2016. [Online]. Available: http://doi.acm.org/10.1145/2930957.2930963
[5] B. T. Loo, T. Condie, M. Garofalakis, D. E. Gay, J. M. Hellerstein, P. Maniatis, R. Ramakrishnan, T. Roscoe, and I. Stoica, "Declarative networking," Commun. ACM, vol. 52, no. 11, pp. 87-95, Nov. 2009. [Online]. Available: http://doi.acm.org/10.1145/1592761.1592785

[6] A. Wang, P. Basu, B. T. Loo, and O. Sokolsky, "Declarative network verification," in International Symposium on Practical Aspects of Declarative Languages. Springer, 2009, pp. 61-75.

[7] T. L. Hinrichs, N. S. Gude, M. Casado, J. C. Mitchell, and S. Shenker, "Practical declarative network management," in Proceedings of the 1st ACM Workshop on Research on Enterprise Networking, ser. WREN '09. New York, NY, USA: ACM, 2009, pp. 1-10. [Online]. Available: http://doi.acm.org/10.1145/1592681.1592683

[8] N. P. Lopes, J. A. Navarro, A. Rybalchenko, and A. Singh, "Applying prolog to develop distributed systems," Theory and Practice of Logic Programming, vol. 10, no. 4-6, pp. 691-707, 2010.

[9] H. T. Lim, D. Herrscher, and F. Chaari, "Performance comparison of ieee $802.1 \mathrm{q}$ and ieee $802.1 \mathrm{avb}$ in an ethernet-based in-vehicle network," in Computing Technology and Information Management (ICCM), 2012 8th International Conference on, vol. 1, April 2012, pp. 1-6.

[10] G. Alderisi, A. Caltabiano, G. Vasta, G. Iannizzotto, T. Steinbach, and L. L. Bello, "Simulative assessments of ieee 802.1 ethernet avb and time-triggered ethernet for advanced driver assistance systems and in-car infotainment," in Vehicular Networking Conference (VNC), 2012 IEEE, Nov 2012, pp. 187-194.

[11] P. Meyer, T. Steinbach, F. Korf, and T. C. Schmidt, "Extending ieee 802.1 avb with time-triggered scheduling: A simulation study of the coexistence of synchronous and asynchronous traffic," in 2013 IEEE Vehicular Networking Conference, Dec 2013, pp. 47-54.

[12] T. Steinbach, H. T. Lim, F. Korf, T. C. Schmidt, D. Herrscher, and A. Wolisz, "Beware of the hidden! how cross-traffic affects quality assurances of competing real-time ethernet standards for in-car communication," in Local Computer Networks (LCN), 2015 IEEE 40th Conference on, Oct 2015, pp. 1-9.

[13] S. Thangamuthu, N. Concer, P. J. L. Cuijpers, and J. J. Lukkien, "Analysis of ethernet-switch traffic shapers for in-vehicle networking applications," in 2015 Design, Automation Test in Europe Conference Exhibition (DATE), March 2015, pp. 55-60.

[14] D. Thiele, R. Ernst, and J. Diemer, "Formal worst-case timing analysis of ethernet tsn's time-aware and peristaltic shapers," in Vehicular Networking Conference (VNC), 2015 IEEE, Dec 2015, pp. 251-258.

[15] G. Alderisi, G. Iannizzotto, and L. Lo Bello, "Towards ieee 802.1 ethernet avb for advanced driver assistance systems: a preliminary assessment," Proceedings of 2012 IEEE 17th International Conference on Emerging Technologies \& Factory Automation (ETFA 2012), p. 4, 2012. 